\title{
37. ORGANIC GEOCHEMISTRY OF UPPER JURASSIC-CRETACEOUS SEDIMENTS FROM SITE 511, LEG 71, WESTERN SOUTH ATLANTIC ${ }^{1}$
}

\author{
G. Deroo, J. P. Herbin, and J. Roucaché, Institut Français du Pétrole, Paris, France
}

\begin{abstract}
Cretaceous and Jurassic sediments $435 \mathrm{~m}$ thick were drilled at Site 511, in the basin province of the Falkland Plateau, during DSDP Leg 71.

The calcareous Unit 3 and the clayey zeolitic Unit 4, both of Senonian age, revealed poorly preserved organic matter indicative of oxidized environments. The same characteristics prevailed for the clayey Unit 5 of Turonian to Albian age. Strictly reducing environments existed for black facies along Unit 6 of earliest Albian to Late Jurassic age and allowed the preservation of a rich organic material that is marine in origin. Besides the transition from reducing conditions in Unit 6 to oxidizing conditions in Unit 5, there are 20 meters of sediments in Cores 56-58 where detrital, nonmarine and then marine organic matter, both implying more or less reducing environments, are interlain by poorly preserved material. In the black shales of the bottom Cores 69 and 70 , some nonmarine detritus is mixed with the predominantly marine organic material.

An immature stage of evolution can be assigned to all of the samples studied.

The chapter also undertakes a comparison with contemporaneous lithologies at adjacent Sites 327 and 330 and attempts some reconstruction of the geography of the eastern Falkland Plateau during the Mesozoic.
\end{abstract}

\section{INTRODUCTION}

The study geochemically characterizes the organic matter present in the Cretaceous and Jurassic sediments penetrated during Leg 71 at DSDP Site 511 (Fig. 1).

Pyrolysis assay and carbon analysis were conducted first on 197 samples. Then hydrocarbon extraction and a humic extraction were undertaken for 22 samples and 9 kerogen concentrates were prepared and examined.

Two sets of samples were obtained: (1) during or after the Leg 71 cruise, 173 samples of only a few grams each were collected from sediments ranging from 388 to 629 meters in depth; (2) 24 larger samples representing sediments ranging from 210 to 627 meters in depth, were issued by the Organic Geochemistry Panel.

The sampling was representative of the Cretaceous and Jurassic Lithologic Units 3-6. Unit 3 consists of calcareous ooze and zeolitic calcareous ooze and Unit 4 of gray zeolitic clays, zeolitic claystones, and claystones; both are of Senonian age. Unit 5 consists of variegated claystones, nannofossil claystones, and chalks, mainly of Albian age. Unit 6, ranging in age from early Albian to Late Jurassic, consists of nannofossil mudstones and black mudstones and shales.

\section{ANALYTICAL METHODS}

The analytical procedures are outlined in Figure 2. Organic carbon contents were determined for acid-treated samples with a LECO apparatus. A pyrolysis assay of raw samples was conducted using RockEval techniques (Espitalié et al., 1977). Selected samples were extracted with chloroform and the hydrocarbons were analyzed by gas chromatography and mass spectrometry. The humic fraction was then isolated and its carbon content analyzed with a Carmograph Wosthoff apparatus before the kerogen was prepared (Huc et al., 1978). Elemental analysis was performed on the kerogen concentrate.

${ }^{1}$ Ludwig, W. J., Krasheninnikov, V. A., et al., Init. Repts. DSDP, 71: Washington (U.S. Govt. Printing Office).

\section{RESULTS}

\section{Mineral Carbon and Carbonates (Table 1)}

A low mineral carbon content (0.2-2.1 wt. \%) equivalent to $2-17 \%$ calcium carbonate defines Unit 6, except for the 30-68\% carbonate found in Samples 511-65-2, $112-146 \mathrm{~cm}$. Enrichments of $60 \%$ and more also occur in the intervals from Samples 511-60-5, $51 \mathrm{~cm}$ to $511-$ $59-4,21 \mathrm{~cm}$ and in Sample 511-57-6, 13-21 cm.

Unit 5, with $20-50 \%$ carbonate, represents a relatively carbonate-rich series, compared to the adjacent, carbonate-poor Units 4 and 6.

\section{Organic Carbon (Table 1)}

In Unit 6 , a high organic carbon $\left(\mathrm{C}_{\text {org }}\right)$ content (3-8 wt.\%) is found in the bottom interval, from Sample 511-70-5, $56 \mathrm{~cm}$ to Sample 511-58-4, $105 \mathrm{~cm}$.

Some impoverished layers appear near the top of the unit. They are distributed in two sets: the lower one, from Sample 511-60-5, $51 \mathrm{~cm}$ to Sample 511-59-4, 21 $\mathrm{cm}$, has a $\mathrm{C}_{\text {org }}$ range of $1.0-2.0 \%$ and corresponds to the previously mentioned calcareous interval; the upper one, from Sample 511-59-3, $100 \mathrm{~cm}$ to Sample 511-58-4, $105 \mathrm{~cm}$, has $\mathrm{C}_{\text {org }}$ contents of $0.4-1.9 \%$ alternating with richer ones $(4.2-5.8 \%)$.

At the top of Unit 6 and the bottom of Unit 5, between Samples 511-58-4, $89 \mathrm{~cm}$ and 511-56-4, $4 \mathrm{~cm}$, poor to medium $\mathrm{C}_{\text {org }}$ contents $(0.4-0.9 \%$ ) predominate. A very lean material with $C_{\text {org }}$ contents of $0.1 \%$ and less is found for the most part of Unit 5, and a low to medium $\mathrm{C}_{\text {org }}$ content, from $0.1-0.8 \%$, is characteristic of Unit 4.

\section{Pyrolysis Assay on Raw Samples (Table 1, Fig. 3)}

The method of pyrolysis described in Espitalié et al. (1977), using a Rock-Eval apparatus, allows us to define three types of kerogen (Tissot et al., 1974), which can be 


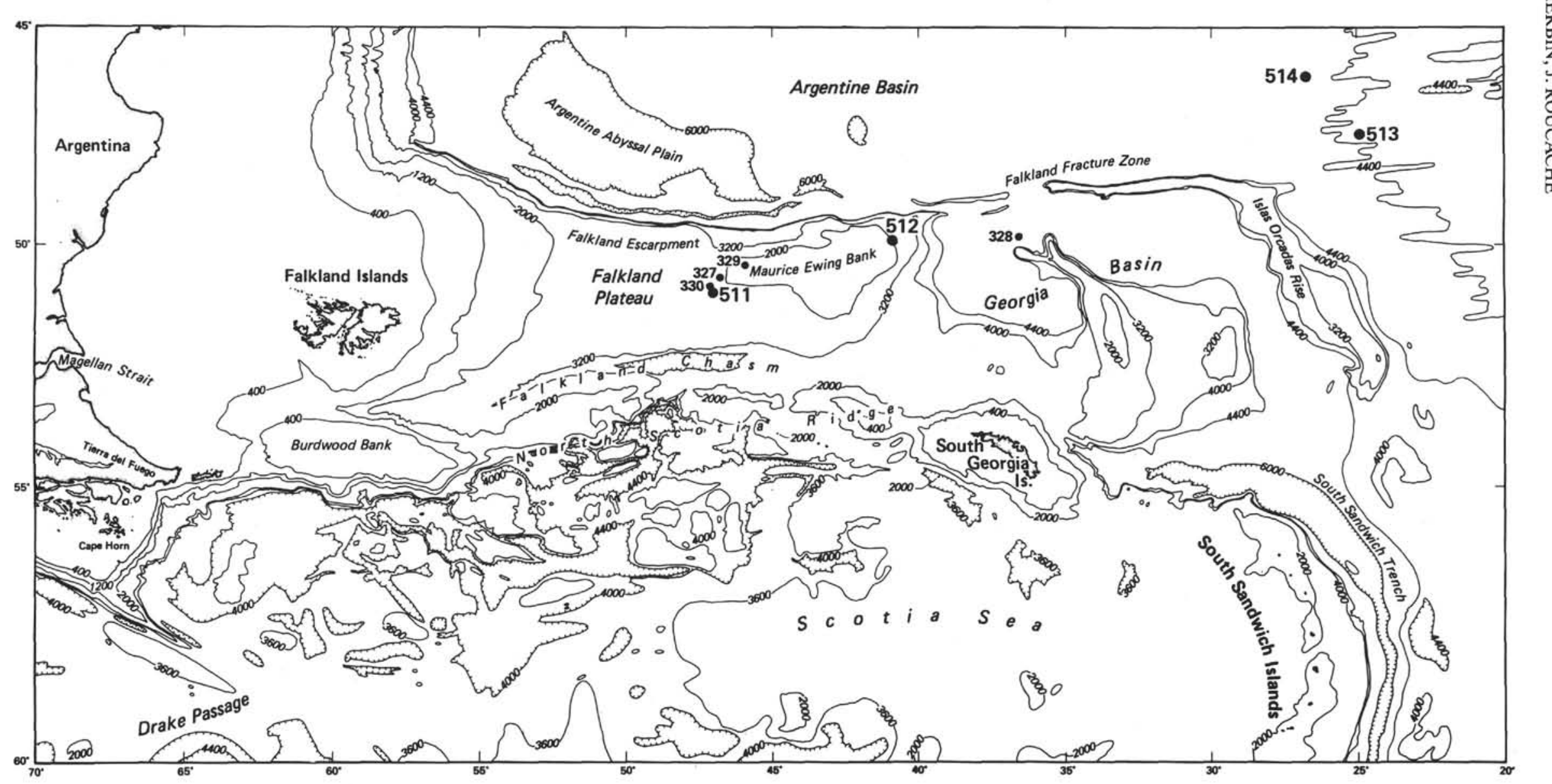

Figure 1. Location of DSDP Site 511. 


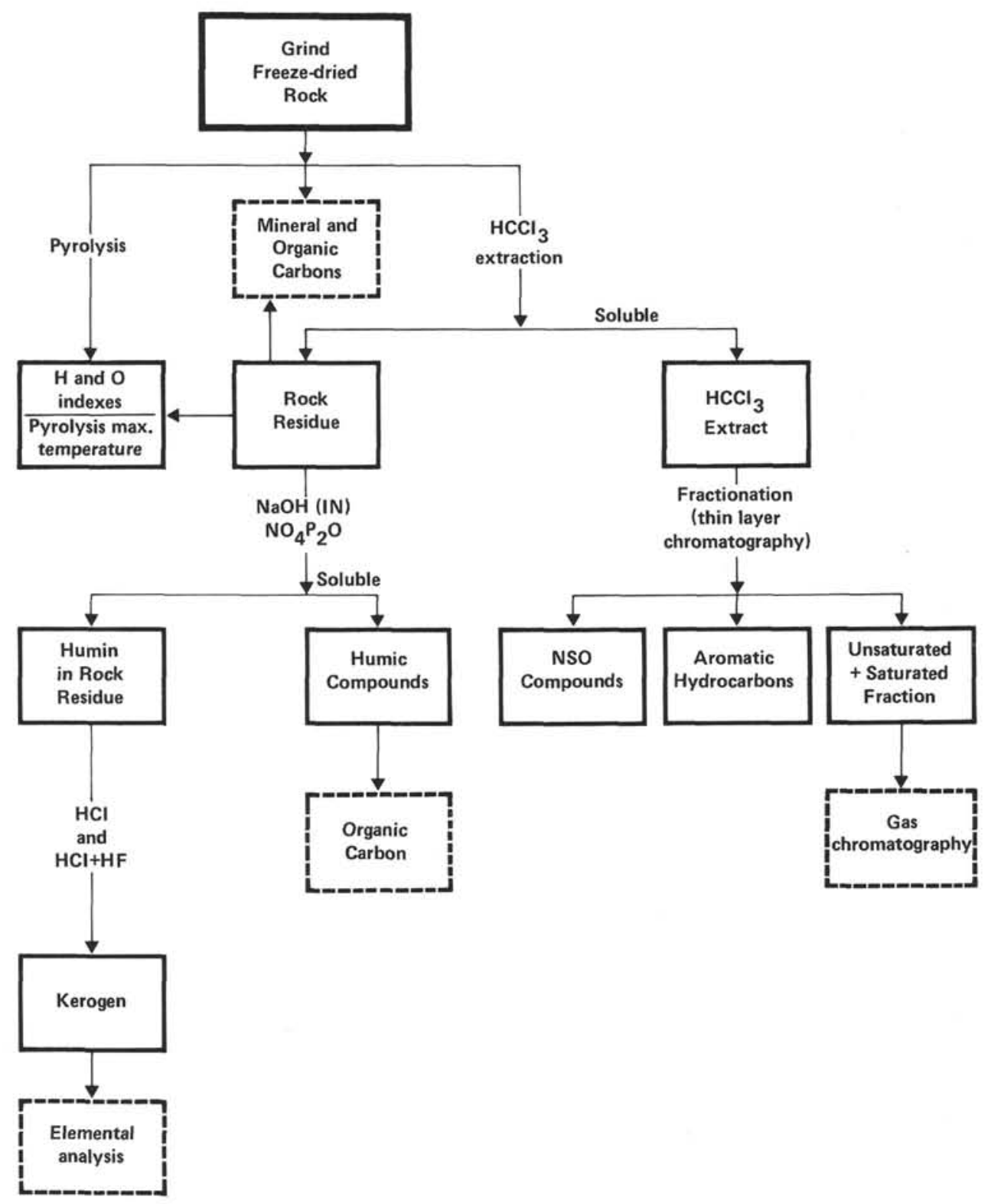

Figure 2. Schematic analytical procedures.

used as references for the characterization of organic matter in ancient sediments. The experimental temperature observed at the top of the pyrolysis peak (Table 1) during this analysis is indicative of the maturity of the organic matter. The temperature corresponding to the immature zone for oil generation is in the range 400 $435^{\circ} \mathrm{C}$, the main oil or mature zone ranges from 435 to $460^{\circ} \mathrm{C}$, and the cracking or gas zone is above $460^{\circ} \mathrm{C}$.

Samples from Site 511 can be assigned to the immature zone, as maximum temperatures were below $425^{\circ} \mathrm{C}$ even for the deeper samples. Some higher temperatures observed in Cores 57 and 58 are due to reworked, more mature material. A progressive increase of temperature ranges is observed along the sequence (Fig. 4) when successive increments of $5^{\circ} \mathrm{C}$ each are considered:

1) $410-414^{\circ} \mathrm{C}$ in Cores 56 to $64,490-575$ meters depth;
2) $415-419^{\circ} \mathrm{C}$ in Cores 65 to $67,575-603$ meters depth;

3) $420-424^{\circ} \mathrm{C}$ for the deeper Cores 68 to $70,603-632$ meters depth.

When the diagram of the hydrogen index $(\mathrm{HI})$ versus the oxygen index.(OI) is examined (Fig. 3A-D), the bulk of the samples is clustered along the kerogen Type I and II reference paths. It corresponds to immature organic matter with a marine origin. As higher maturation cannot be invoked to explain the relatively low HI for the deepest Cores 69 and 70 (Fig. 3D), some dilution with nonmarine organic matter should be considered. All these marine materials reveal medium to high organic carbon contents. Few data points (Cores $37,56,58$ ) are below the Type III reference path (Fig. 3A). Their OI range (100-180) corresponds to immature material with a continental origin, and the low HI (54 and less) implies 


\section{G. DEROO, J. P. HERBIN, J. ROUCACHÉ}

Table 1. Sample information; carbon and pyrolysis assay data, Hole 511.

\begin{tabular}{|c|c|c|c|c|c|c|c|c|c|}
\hline $\begin{array}{l}\text { Lithologic } \\
\text { Unit }\end{array}$ & $\begin{array}{l}\text { Core/Section } \\
\text { (interval in cm) }\end{array}$ & $\begin{array}{c}\text { Depth below } \\
\text { Seafloor } \\
(\mathrm{m})\end{array}$ & $\begin{array}{l}\text { Mineral } \\
\text { Carbon } \\
\text { (wt.\%) }\end{array}$ & $\begin{array}{l}\text { Organic } \\
\text { Carbon } \\
\text { (wt. \%) }\end{array}$ & $\begin{array}{l}\text { Hydrogen } \\
\text { Index } \\
\text { (mg hydroc. } \\
\text { compounds/ } \\
\mathrm{g}_{\text {org }} \text { ) }\end{array}$ & $\begin{array}{c}\text { Oxygen } \\
\text { Index } \\
\text { (mg COO } \\
\mathrm{C}_{\text {org }} \text { ) }\end{array}$ & $\begin{array}{l}\text { Hydrogen } \\
\quad \text { Index } \\
\text { (mg hydroc. } \\
\text { compounds/ } \\
\text { g rock) }\end{array}$ & $\begin{array}{c}\text { Oxygen } \\
\text { Index } \\
\text { (mg CO } \mathrm{CO}_{2} / \\
\mathrm{g} \text { rock) }\end{array}$ & $\begin{array}{c}\text { Pyrolysis } \\
\text { Temperature } \\
\left({ }^{\circ} \mathrm{C}\right)\end{array}$ \\
\hline Unit 4 & $24-4,130-140$ & 210.30 & 7.1 & 0.19 & & & & & \\
\hline & $28-5,130-140$ & 230.80 & 1.1 & 0.24 & 0 & 708 & 0.00 & 1.70 & \\
\hline & $31-5,130-140$ & 259.30 & 0.5 & 0.65 & 0 & 312 & 0.00 & 2.03 & \\
\hline & $34-5,130-140$ & 287.80 & 2.2 & 0.73 & 11 & 295 & 0.08 & 2.15 & 421 \\
\hline & $37-1,130-140$ & 310.30 & 1.2 & 0.84 & 12 & 298 & 0.10 & 2.50 & 421 \\
\hline & $40-4,130-140$ & 343.30 & 0.9 & 0.68 & 9 & 324 & 0.06 & 2.20 & 413 \\
\hline Unit 5 & $43-4,130-140$ & 371.80 & 0.8 & 0.55 & 7 & 438 & 0.04 & 2.41 & 426 \\
\hline & $45-3,94$ & 388.94 & 0.7 & 0.07 & & & & & \\
\hline & $46-2,130-140$ & 397.30 & 0.3 & 0.37 & 0 & 416 & 0.00 & 1.54 & \\
\hline & $46-3,50$ & 399.50 & 1.0 & 0.44 & 0 & 611 & 0.00 & 2.69 & \\
\hline & $47-4,38$ & 408.88 & 1.8 & 0.12 & & & & & \\
\hline & $48-3,38$ & 416.88 & 0.3 & 0.05 & & & & & \\
\hline & $49-4,130-140$ & 428.80 & 1.4 & 0.07 & & & & & \\
\hline & $49-5,23$ & 429.23 & 1.5 & 0.02 & & & & & \\
\hline & $\begin{array}{l}50-3,23 \\
51-1,140\end{array}$ & $\begin{array}{l}435.73 \\
443.40\end{array}$ & $\begin{array}{l}3.8 \\
4.7\end{array}$ & $\begin{array}{l}0.02 \\
0.06\end{array}$ & & & & & \\
\hline & $51-1,146-148$ & 443.46 & 3.3 & 0.06 & & & & & \\
\hline & $51-5,90$ & 446.90 & 6.2 & 0.04 & & & & & \\
\hline & $52-5,130-140$ & 458.80 & 4.5 & 0.07 & & & & & \\
\hline & $52-6,25$ & 459.25 & 5.7 & 0.05 & & & & & \\
\hline & $53-4,100$ & 466.50 & 4.8 & 0.06 & & & & & \\
\hline & $54-2,25$ & 472.25 & 3.0 & 0.11 & & & & & \\
\hline & $54-5,125$ & 476.25 & 2.5 & 0.06 & & & & & \\
\hline & $55-2,42$ & 481.92 & 3.4 & 0.12 & & & & & \\
\hline & $55-4,130-140$ & 485.80 & 4.3 & 0.07 & & & & & \\
\hline & $55-5,42$ & 486.42 & 0.3 & 0.05 & & & & & \\
\hline & $56-2,71$ & 491.71 & 2.8 & 0.90 & 0 & 226 & 0.00 & 2.03 & \\
\hline & $56-3,145-147$ & 493.45 & 0.4 & 0.14 & & & & & \\
\hline & $56-4,04-62$ & 493.54 & 0.2 & 0.44 & 34 & 107 & 0.15 & 0.47 & 415 \\
\hline & $56-4,04-06$ & 493.54 & 0.3 & 0.45 & 39 & 173 & 0.17 & 0.78 & 406 \\
\hline & $56-4,22-24$ & 493.72 & 0.3 & 0.43 & 40 & 151 & 0.17 & 0.65 & 414 \\
\hline & $56-4,38-41$ & 493.88 & 0.1 & 0.43 & 48 & 144 & 0.21 & 0.62 & 414 \\
\hline & $56-4,60-62$ & 494.10 & 0.2 & 0.43 & 34 & 123 & 0.14 & 0.53 & 414 \\
\hline & $56-4,71-110$ & 494.21 & 1.1 & 0.13 & & & & & \\
\hline & $56-4,71$ & 494.21 & 0.3 & 0.17 & & & & & \\
\hline & $56-4,76-78$ & 494.26 & 0.3 & 0.13 & & & & & \\
\hline & $56-4,108-110$ & 494.58 & 0.3 & 0.15 & & & & & \\
\hline & $56-5,03-05$ & 495.03 & 3.0 & 0.11 & & & & & \\
\hline & $56-5,20-22$ & 495.20 & 0.6 & 0.33 & 29 & 570 & 0.10 & 1.88 & 421 \\
\hline Unit 6 & $56-5,80-82$ & 495.80 & 0.2 & 0.57 & 41 & 125 & 0.23 & 0.71 & 416 \\
\hline & $56-5,130-132$ & 496.30 & 0.2 & 0.61 & 50 & 167 & 0.31 & 1.02 & \\
\hline & $56-5,146-148$ & 496.46 & 0.4 & 0.51 & 53 & 125 & 0.27 & 0.64 & 413 \\
\hline & $57-1,20-22$ & 499.20 & 0.6 & 0.49 & 30 & 133 & 0.15 & 0.65 & 407 \\
\hline & $57-1,75-77$ & 499.75 & 0.5 & 0.48 & 48 & 129 & 0.22 & 0.62 & 407 \\
\hline & $57-1,124-126$ & 500.24 & 0.5 & 0.80 & 56 & 156 & 0.45 & 1.25 & 416 \\
\hline & $57-2,15-17$ & 500.65 & 0.4 & 0.57 & 41 & 119 & 0.23 & 0.68 & 418 \\
\hline & $57-2,35$ & 500.85 & 0.6 & 0.75 & 29 & 120 & 0.22 & 0.90 & 418 \\
\hline & $57-2,95-97$ & 501.45 & 0.6 & 2.28 & 167 & 117 & 3.80 & 2.66 & 430 \\
\hline & $57-3,37-38$ & 502.37 & 1.0 & 0.86 & 75 & 336 & 0.65 & 2.89 & 422 \\
\hline & $57-3,110-112$ & 503.10 & 0.4 & 0.49 & 33 & 216 & 0.16 & 1.06 & 411 \\
\hline & $57-4,00-02$ & 503.50 & 1.4 & 0.24 & 44 & 996 & 0.11 & 2.39 & 414 \\
\hline & $57-4,03-05$ & 503.53 & 0.4 & 0.96 & 273 & 140 & 2.62 & 1.34 & \\
\hline & $57-4,07-09$ & 503.57 & 0.2 & 3.75 & 504 & 47 & 18.91 & 1.78 & 398 \\
\hline & $57-4,14-16$ & 503.64 & 0.5 & 0.33 & 56 & 336 & 0.19 & 1.11 & 414 \\
\hline & $57-4,18-20$ & 503.68 & 1.9 & 0.16 & & & & & \\
\hline & $57-4,26-28$ & 503.76 & 0.4 & 0.24 & 47 & 471 & 0.11 & 1.13 & 404 \\
\hline & $57-4,90-92$ & 504.40 & 0.6 & 0.93 & 31 & 151 & 0.29 & 1.40 & 414 \\
\hline & $57-5,78$ & 505.78 & 0.7 & 0.55 & 83 & 540 & 0.46 & 2.97 & 427 \\
\hline & $57-5,98-100$ & 505.98 & 0.3 & 0.18 & & & & & \\
\hline & $57-5,104-106$ & 506.04 & 1.5 & 1.09 & 153 & 194 & 1.67 & 2.11 & 431 \\
\hline & $57-5,144-146$ & 506.44 & 0.5 & 0.57 & 43 & 267 & 0.24 & 1.52 & 418 \\
\hline & $57-6,06-08$ & 506.56 & 0.8 & 0.91 & 104 & 187 & 0.94 & 1.70 & 421 \\
\hline & $57-6,13-15$ & 506.63 & 6.9 & 0.17 & & & & & \\
\hline & $57-6,19-21$ & 506.69 & 6.9 & 0.11 & & & & & \\
\hline & $58-1,38$ & 508.88 & 0.4 & 0.45 & 20 & 209 & 0.09 & 0.94 & 407 \\
\hline & $58-3,38$ & 511.88 & 0.7 & 0.38 & 38 & 400 & 0.14 & 1.52 & 414 \\
\hline & $58-3,110-112$ & 512.60 & 0.6 & 0.31 & 46 & 377 & 0.14 & 1.17 & 416 \\
\hline & $58-3,116-118$ & 512.66 & 1.3 & 0.14 & & & & & \\
\hline & $58-3,130-140$ & 512.80 & 0.3 & 0.31 & 28 & 361 & 0.09 & 1.12 & 416 \\
\hline & $58-4,02-04$ & 513.02 & 0.3 & 0.24 & 28 & 283 & 0.07 & 0.68 & 430 \\
\hline & $58-4,28-49$ & 513.28 & 0.1 & 0.38 & 54 & 163 & 0.20 & 0.62 & 411 \\
\hline & $58-4,28-30$ & 513.28 & 0.2 & 0.47 & 44 & 147 & 0.21 & 0.69 & 404 \\
\hline & $58-4,47-49$ & 513.47 & 0.3 & 0.35 & 38 & 146 & 0.13 & 0.51 & 411 \\
\hline & $58-4,53-89$ & 513.53 & 0.4 & 0.13 & & & & & \\
\hline & $58-4,53-55$ & 513.53 & 0.3 & 0.15 & & & & & \\
\hline & $58-4,59-61$ & 513.59 & 0.3 & 0.07 & & & & & \\
\hline & $58-4,71-73$ & 513.71 & 0.4 & 0.06 & & & & & \\
\hline & $58-4,87-89$ & 513.87 & 1.4 & 0.17 & & & & & \\
\hline & $58-4,105-107$ & 514.05 & 0.7 & 6.10 & 366 & 35 & 22.33 & 2.12 & 414 \\
\hline & $59-1,26-28$ & 518.26 & 3.4 & 6.24 & 502 & 54 & 31.31 & 3.39 & 411 \\
\hline & $59-1,100-102$ & 519.00 & 0.6 & 5.32 & 371 & 33 & 19.76 & 1.78 & 415 \\
\hline & $59-2,28-30$ & 519.78 & 0.7 & 1.70 & 91 & 146 & 1.55 & 2.48 & 426 \\
\hline & $59-2,81-83$ & 520.31 & 2.1 & 4.17 & 316 & 66 & 13.17 & 2.76 & 420 \\
\hline & $59-3,10-12$ & 521.10 & 1.8 & 5.29 & 413 & 55 & 21.83 & 2.91 & 413 \\
\hline & $59-3,60-62$ & 521.60 & 1.6 & 5.69 & 299 & 49 & 17.01 & 2.80 & 407 \\
\hline & $59-3,79-82$ & 521.79 & 0.3 & 0.48 & 138 & 200 & 0.66 & 0.96 & 409 \\
\hline & $59-3,79-80$ & 521.79 & 0.4 & 0.55 & 171 & 242 & 0.94 & 1.33 & 419 \\
\hline & $59-3,81-82$ & 521.81 & 0.3 & 0.44 & 107 & 225 & 0.47 & 0.99 & 411 \\
\hline & $59-3,83-95$ & 521.83 & 2.1 & 5.75 & 374 & 45 & 21.48 & 2.57 & 415 \\
\hline
\end{tabular}


Table 1. (Continued).

\begin{tabular}{|c|c|c|c|c|c|c|c|c|}
\hline $\begin{array}{c}\text { Lithologic } \\
\text { Unit }\end{array}$ & $\begin{array}{c}\text { Core/Section } \\
\text { (interval in cm) }\end{array}$ & $\begin{array}{l}\text { Depth below } \\
\text { Seafloor } \\
(\mathrm{m})\end{array}$ & $\begin{array}{l}\text { Mineral } \\
\text { Carbon } \\
\text { (wt.\%) }\end{array}$ & $\begin{array}{l}\text { Organic } \\
\text { Carbon } \\
\text { (wt.\%) }\end{array}$ & $\begin{array}{l}\text { Hydrogen } \\
\text { Index } \\
\text { (mg hydroc. } \\
\text { compounds/ } \\
8 \text { C }_{\text {org) }}\end{array}$ & $\begin{array}{c}\text { Oxygen } \\
\text { Index } \\
\left(\mathrm{mg} \mathrm{CO}_{2} /\right. \\
\left.\mathrm{C}_{\mathrm{org}}\right)\end{array}$ & $\begin{array}{l}\text { Hydrogen } \\
\text { Index } \\
\text { (mg hydroc. } \\
\text { compounds/ } \\
8 \text { rock) }\end{array}$ & $\begin{array}{c}\text { Oxygen } \\
\text { Index } \\
\text { (mg CO} 2 \text { ' } \\
\text { g rock) }\end{array}$ \\
\hline Unit 6 & $59-3,83-95$ & 521.83 & 1.6 & 5.06 & 410 & 64 & 20.72 & 3.25 \\
\hline & $59-3,89-91$ & 521.89 & 2.7 & 4.97 & 445 & 58 & 22.10 & 2.90 \\
\hline & $59-3,93-95$ & 521.93 & 2.5 & 5.85 & 479 & 52 & 28.02 & 3.06 \\
\hline & $59-3,98-100^{\circ}$ & 521.98 & 3.6 & 1.92 & 309 & 107 & 5.94 & 2.05 \\
\hline & $59-3,98-100$ & 521.98 & 2.0 & 2.17 & 324 & 113 & 7.04 & 2.45 \\
\hline & $59-4,21-22$ & 522.71 & 7.5 & 1.96 & 404 & 79 & 7.91 & 1.54 \\
\hline & $59-4,25-27$ & 522.75 & 3.5 & 5.20 & 506 & 58 & 26.34 & 3.02 \\
\hline & $59-4,29-31$ & 522.79 & 5.0 & 2.22 & 293 & 105 & 6.51 & 2.32 \\
\hline & $59-4,36-38$ & 522.86 & 3.5 & 5.69 & 518 & 63 & 29.46 & 3.61 \\
\hline & $59-4,45-47$ & 522.95 & 10.2 & 1.36 & 479 & 91 & 6.52 & 1.24 \\
\hline & $59-4,53-54$ & 523.03 & 7.7 & 2.50 & 426 & 74 & 10.66 & 1.85 \\
\hline & $59-4,60-62$ & 523.10 & 9.8 & 2.01 & 494 & 75 & 9.92 & 1.50 \\
\hline & $59-4,74-76$ & 523.24 & 1.1 & 1.14 & 234 & 150 & 2.67 & 1.71 \\
\hline & $59-4,122-124$ & 523.72 & 3.8 & 5.32 & 474 & 55 & 25.20 & 2.91 \\
\hline & $60-1,00-02$ & 527.50 & 3.3 & 6.08 & 415 & 63 & 25.20 & 3.84 \\
\hline & $60-1,105-107$ & 528.55 & 3.0 & 3.76 & 394 & 75 & 14.83 & 2.81 \\
\hline & $60-2,52-54$ & 529.52 & 3.4 & 4.83 & 451 & 57 & 21.79 & 2.76 \\
\hline & $60-2,136-137$ & 530.36 & 10.3 & 1.04 & 350 & 80 & 3.64 & 0.83 \\
\hline & $60-3,06-08$ & 530.56 & 1.9 & 5.02 & 251 & 64 & 12.60 & 3.23 \\
\hline & $60-3,18$ & 530.68 & 2.5 & 4.65 & 236 & 100 & 10.95 & 4.67 \\
\hline & $60-3,57-59$ & 531.07 & 4.4 & 5.20 & 394 & 56 & 20.46 & 2.92 \\
\hline & $60-4,53-55$ & 532.53 & 3.4 & 2.98 & 326 & 82 & 9.71 & 2.44 \\
\hline & $60-4,113-115$ & 533.13 & 3.8 & 4.72 & 352 & 56 & 16.61 & 2.62 \\
\hline & $60-5,49-51$ & 533.99 & 7.2 & 1.65 & 260 & 94 & 4.29 & 1.55 \\
\hline & $60-5,102-104$ & 534.52 & 0.3 & 3.47 & 424 & 28 & 14.70 & 0.98 \\
\hline & $60-5,130-140$ & 534.80 & 0.3 & 5.28 & 405 & 19 & 21.38 & 0.99 \\
\hline & $60-6,05-07$ & 535.05 & 0.4 & 3.82 & 428 & 24 & 16.34 & 0.90 \\
\hline & $60-6,18$ & 535.18 & 0.1 & 2.73 & 583 & 32 & 15.92 & 0.87 \\
\hline & $61-2,80$ & 539.30 & 1.4 & 4.98 & 438 & 80 & 21.82 & 4.00 \\
\hline & $61-4,73-75$ & 542.23 & 0.4 & 5.10 & 498 & 34 & 25.37 & 1.74 \\
\hline & $61-4,92-94$ & 542.42 & 0.8 & 6.52 & 552 & 38 & 36.02 & 2.48 \\
\hline & $61-4,100-102$ & 542.50 & 3.5 & 4.32 & 552 & 55 & 23.83 & 2.36 \\
\hline & $61-4,136-138$ & 542.86 & 1.8 & 4.33 & 542 & 55 & 23.45 & 2.36 \\
\hline & $61-4,140$ & 542.90 & 1.8 & 4.52 & 565 & 66 & 25.52 & 2.97 \\
\hline & $61-5,08-10$ & 543.08 & 2.9 & 4.27 & 572 & 57 & 24.42 & 2.45 \\
\hline & $61-5,11-12$ & 543.11 & 3.2 & 3.66 & 537 & 78 & 19.66 & 2.87 \\
\hline & $61-5,15-17$ & 543.15 & 2.5 & 3.84 & 542 & 73 & 20.82 & 2.82 \\
\hline & $61-5,45-47$ & 543.45 & 1.3 & 4.74 & 501 & 49 & 23.72 & 2.32 \\
\hline & $61-5,70-72$ & 543.70 & 1.4 & 4.73 & 544 & 50 & 25.74 & 2.38 \\
\hline & $62-1,48-50$ & 546.98 & 1.1 & 5.50 & 555 & 51 & 30.52 & 2.80 \\
\hline & $62-1,70-72$ & 547.20 & 1.5 & 5.75 & 566 & 55 & 32.55 & 3.15 \\
\hline & $62-1,89-91$ & 547.39 & 1.3 & 4.36 & 535 & 55 & 23.34 & 2.38 \\
\hline & $62-1,96-98$ & 547,46 & 0.5 & 4.04 & 448 & 45 & 18.11 & 1.82 \\
\hline & $62-1,96-134$ & 547.56 & 0.4 & 5.10 & 559 & 40 & 28.52 & 2.04 \\
\hline & $62-1,112-114$ & 547.62 & 0.5 & 8.17 & 593 & 37 & 48.46 & 3.03 \\
\hline & $62-1,132-134$ & 547.82 & 0.6 & 5.64 & 509 & 28 & 28.71 & 1.57 \\
\hline & $62-2,02-04$ & 548.02 & 0.2 & 5.55 & 504 & 23 & 27.99 & 1.27 \\
\hline & $62-2,09-10$ & 548.09 & 0.2 & 1.22 & 274 & 29 & 3.34 & 0.35 \\
\hline & $62-2,80$ & 548.80 & 0.2 & 4.00 & 351 & 28 & 14.04 & 1.11 \\
\hline & $62-5,70$ & 553.20 & 1.5 & 5.44 & 504 & 56 & 27.42 & 3.06 \\
\hline & $62-5,130-140$ & 553.80 & 0.3 & 5.25 & 360 & 24 & 18.88 & 1.24 \\
\hline & $63-1,30$ & 554.30 & 0.8 & 3.53 & 355 & 65 & 12.55 & 2.31 \\
\hline & $63-3,70-72$ & 557.70 & 1.2 & 5.59 & 478 & 46 & 26.72 & 2.58 \\
\hline & $63-3,97-99$ & 557.97 & 1.2 & 3.96 & 347 & 48 & 13.72 & 1.92 \\
\hline & $63-3,82-84$ & 558.32 & 0.9 & 3.93 & 413 & 53 & 16.24 & 2.07 \\
\hline & $63-3,08$ & 559.08 & 0.5 & 4.35 & 442 & 52 & 19.23 & 2.27 \\
\hline & $63-3,43-45$ & 559.43 & 0.7 & 4.90 & 478 & 42 & 23.44 & 2.04 \\
\hline & $63-3,56-58$ & 559.56 & 0.9 & 5.75 & 531 & 41 & 30.53 & 2.37 \\
\hline & $63-3,68-70$ & 559.68 & 1.0 & 6.43 & 540 & 40 & 34.70 & 2.60 \\
\hline & $63-3,96-98$ & 559.96 & 0.7 & 4.31 & 444 & 46 & 19.14 & 1.99 \\
\hline & $63-3,108-110$ & 560.08 & 0.5 & 4.14 & 398 & 45 & 16.48 & 1.88 \\
\hline & $63-3,121-123$ & 560.21 & 1.0 & 4.45 & 417 & 44 & 18.57 & 1.95 \\
\hline & $64-1,18-19$ & 565.68 & 0.6 & 4.41 & 427 & 46 & 18.85 & 2.02 \\
\hline & $64-2,18-19$ & 567,18 & 0.7 & 3.34 & 331 & 56 & 11.06 & 1.88 \\
\hline & $64-2,75$ & 567.75 & 1.0 & 3.40 & 333 & 63 & 11.32 & 2.14 \\
\hline & $64-3,18-19$ & 568.68 & 0.6 & 3.82 & 371 & 55 & 14.18 & 2.10 \\
\hline & $64-4,18-19$ & 570.18 & 0.8 & 3.38 & 374 & 56 & 12.63 & 1.89 \\
\hline & $64-4,130-140$ & 571.30 & 1.0 & 5.69 & 426 & 36 & 24.25 & 2.04 \\
\hline & $64-5,18-19$ & 571.68 & 0.7 & 3.57 & 392 & 50 & 14.00 & 1.80 \\
\hline & $64-5,76$ & 572.26 & 1.2 & 4.29 & 447 & 68 & 19.17 & 2.92 \\
\hline & $64-6,18-19$ & 573.18 & 1.4 & 4.42 & 412 & 53 & 18.20 & 2.33 \\
\hline & $65-2,14$ & 576.64 & 0.7 & 4.26 & 397 & 58 & 16.91 & 2.49 \\
\hline & $65-2,78-109$ & 577.28 & 0.8 & 4.85 & 453 & 48 & 21.99 & 2.34 \\
\hline & $65-2,78-80$ & 577.28 & 0.6 & 5.06 & 483 & 44 & 24.43 & 2.24 \\
\hline & $65-2,94-96$ & 577.44 & 1.0 & 4.81 & 472 & 51 & 22.71 & 2.44 \\
\hline & $65-2,106-109$ & 577.56 & 0.8 & 4.75 & 472 & 50 & 22.44 & 2.38 \\
\hline & $65-2,112-114$ & 577.62 & 4.1 & 3.70 & 463 & 51 & 17.14 & 1.90 \\
\hline & $65-2,114-116$ & 577.64 & 7.9 & 2.02 & 463 & 53 & 9.36 & 1.08 \\
\hline & $65-2,118-120$ & 577.68 & 3.5 & 3.78 & 445 & 54 & 16.82 & 2.05 \\
\hline & $65-2,144-146$ & 577.94 & 8.2 & 1.77 & 472 & 62 & 8.36 & 1.10 \\
\hline & $65-2,148-150$ & 577.98 & 1.9 & 4.08 & 441 & 54 & 17.98 & 2.22 \\
\hline & $65-4,122$ & 580.72 & 0.9 & 5.26 & 499 & 51 & 26.26 & 2.68 \\
\hline & $66-1,36-38$ & 584.86 & 1.5 & 3.99 & 401 & 54 & 16.01 & 2.15 \\
\hline & $66-2,32$ & 586.32 & 1.2 & 4.70 & 405 & 54 & 19.05 & 2.55 \\
\hline & $66-2,34-36$ & 586.34 & 0.9 & 4.74 & 432 & 47 & 20.48 & 2.23 \\
\hline & $66-3,36-38$ & 587.86 & 1.3 & 5.17 & 451 & 51 & 23.34 & 2.65 \\
\hline & $66-4,32$ & 589.32 & 0.9 & 5.09 & 458 & 61 & 23.30 & 3.12 \\
\hline & $66-4,34-36$ & 589.34 & 1.2 & 5.51 & 478 & 45 & 26.35 & 2.48 \\
\hline & $66-4,130-140$ & 590.30 & 1.0 & 6.25 & 417 & 33 & 26.07 & 2.09 \\
\hline
\end{tabular}


Table 1. (Continued).

\begin{tabular}{|c|c|c|c|c|c|c|c|c|c|}
\hline $\begin{array}{l}\text { Lithologic } \\
\text { Unit }\end{array}$ & $\begin{array}{c}\text { Core/Section } \\
\text { (interval in cm) }\end{array}$ & $\begin{array}{l}\text { Depth below } \\
\text { Seafloor } \\
\text { (m) }\end{array}$ & $\begin{array}{l}\text { Mineral } \\
\text { Carbon } \\
\text { (wt.\%) }\end{array}$ & $\begin{array}{l}\text { Organic } \\
\text { Carbon } \\
\text { (wt. \%) }\end{array}$ & $\begin{array}{l}\text { Hydrogen } \\
\text { Index } \\
\text { (mg hydroc. } \\
\text { compounds/ } \\
\text { g C Corg) }\end{array}$ & $\begin{array}{c}\text { Oxygen } \\
\text { Index } \\
\text { (mg CO} 2 / \\
\mathrm{C}_{\text {org }} \text { ) }\end{array}$ & $\begin{array}{l}\text { Hydrogen } \\
\text { Index } \\
\text { (mg hydroc. } \\
\text { compounds/ } \\
\text { g rock) }\end{array}$ & $\begin{array}{c}\text { Oxygen } \\
\text { Index } \\
\text { (mg CO} 2 / \\
\text { g rock) }\end{array}$ & $\begin{array}{c}\text { Pyrolysis } \\
\text { Temperature } \\
\left({ }^{\circ} \mathrm{C}\right)\end{array}$ \\
\hline \multirow[t]{28}{*}{ Unt 6} & $66-5,36-38$ & 590.86 & 0.9 & 5.51 & 464 & 47 & 25.57 & 2.60 & 416 \\
\hline & $66-6,34-36$ & 592.34 & 0.8 & 5.36 & 447 & 54 & 23.97 & 2.87 & 414 \\
\hline & $67-1,88-90$ & 594.88 & 0.7 & 5.22 & 455 & 51 & 23.78 & 2.65 & 424 \\
\hline & $67-1,90$ & 594.90 & 0.9 & 5.28 & 445 & 52 & 23.52 & 2.74 & 411 \\
\hline & $67-2,88-90$ & 596.38 & 0.9 & 5.10 & 454 & 54 & 23.14 & 2.77 & 422 \\
\hline & $67-3,88-90$ & 597.88 & 1.6 & 3.91 & 393 & 71 & 15.37 & 2.79 & 424 \\
\hline & $67-4,88-90$ & 599.38 & 0.6 & 5.05 & 422 & 54 & 21.29 & 2.73 & 417 \\
\hline & $67-4,90$ & 599.40 & 0.6 & 4.83 & 429 & 61 & 20.73 & 2.95 & 408 \\
\hline & $67-5,88-90$ & 600.88 & 0.9 & 4.09 & 411 & 62 & 16.83 & 2.53 & 418 \\
\hline & $68-1,23$ & 603.73 & 2.1 & 3.86 & 384 & 63 & 14.81 & 2.43 & 420 \\
\hline & $68-1,58-60$ & 604.08 & 3.5 & 3.27 & 419 & 76 & 13.70 & 2.49 & 423 \\
\hline & $68-2,58-60$ & 605.58 & 1.3 & 3.24 & 326 & 57 & 10.55 & 1.84 & 417 \\
\hline & $68-2,130-140$ & 606.30 & 0.3 & 3.88 & 270 & 30 & 10.49 & 1.15 & 423 \\
\hline & $69-1,36-38$ & 613.36 & 0.3 & 2.85 & 242 & 33 & 6.89 & 0.93 & 424 \\
\hline & $69-2,34$ & 614.86 & 0.4 & 3.14 & 254 & 30 & 7.98 & 0.94 & 420 \\
\hline & $69-2,36-38$ & 614.86 & 0.3 & 3.03 & 269 & 30 & 8.14 & 0.91 & 422 \\
\hline & $69-3,36-38$ & 616.36 & 0.1 & 3.13 & 247 & 26 & 7.73 & 0.82 & 422 \\
\hline & $69-4,34$ & 617.84 & 0.3 & 3.26 & 285 & 27 & 9.29 & 0.89 & 415 \\
\hline & $69-4,36-38$ & 617.86 & 0.4 & 3.33 & 295 & 35 & 9.83 & 1.16 & 411 \\
\hline & 69-5, 36-38 & 619.36 & 0.2 & 4.30 & 342 & 25 & 14.70 & 1.09 & 417 \\
\hline & $70-1,54-56$ & 623.04 & 0.3 & 4.04 & 326 & 25 & 13.18 & 1.03 & 424 \\
\hline & $70-2,54-56$ & 624.54 & 0.6 & 3.78 & 297 & 30 & 11.24 & 1.12 & 420 \\
\hline & $70-2,68$ & 624.68 & 0.2 & 1.89 & 210 & 35 & 3.97 & 0.67 & 413 \\
\hline & $70-3,54-56$ & 626.04 & 0.3 & 4.42 & 356 & 27 & 15.74 & 1.19 & 411 \\
\hline & $70-3,130-140$ & 626.80 & 0.3 & 4.91 & 348 & 37 & 17.07 & 1.81 & 421 \\
\hline & $70-4,54-56$ & 627.54 & 0.3 & 4.04 & 358 & 28 & 14.46 & 1.15 & 420 \\
\hline & $70-4,68$ & 627.68 & 0.3 & 4.54 & 376 & 27 & 17.07 & 1.24 & 424 \\
\hline & $70-5,54-56$ & 629.04 & 0.3 & 4.08 & 305 & 26 & 12.43 & 1.06 & 417 \\
\hline
\end{tabular}

- and 59-4, 21-22.

Note: Blanks in the pyrolysis columns indicate $\mathrm{C}_{\text {org }}$ contents of $0.20 \%$ and less.

some alteration of this organic matter. Some other points are far off the reference paths. They correspond to poor organic carbon contents. These samples probably consist of "undifferentiated" or residual material (Tissot et al., 1979). A last group of points clusters between paths II and III (Fig. 3B); their OI increases from 50 to 200 as their HI decreases from 400 to 100 . Two explanations can be proposed for the trend, which represents either a Type II organic matter mixed with residual material, the latter tending to decrease the $\mathrm{HI}$ and to increase the OI, or a Type II material enriched with oxygenated compounds (OI) at the expense of the hydrogenated $(\mathrm{HI})$ ones. The second explanation is supported by the kerogen data, as will be shown later.

\section{Humic Compounds (Table 2)}

Poor yields of humic compounds were found for most of the 21 samples selected for humic fractionation; they are below $50 \mathrm{ppm}$ in content, which can be considered as the lower limit of significance. Low contents of total organic carbon explain the major part of these insignificant data. The other low yields are observed for the black mudstone facies, which are rich in organic matter. Even for the relatively higher content of humic carbon versus total carbon (8-12 wt. \%) found for three samples of Unit 4, the humic fraction is subordinate so that it can be considered that the kerogen fraction next examined is the most representative fraction of the total organic matter.

\section{Kerogen Fraction (Table 3)}

Nine of the previously selected samples were prepared. Elemental analysis of the kerogen concentrate reveals a large content of pyrite (24-45 wt.\%), except for Sample 511-56-4, $4 \mathrm{~cm}$, which is representative of the black shale facies at the bottom of Unit 5, where black shales are interlain with muddy chalk and zeolitic claystones. The data are plotted on a Van Krevelen diagram (Fig. 5) and are comparable to the three reference evolution paths for Types I, II, and III kerogens of ancient sediments (Tissot et al., 1974). An immature stage can be assigned to the samples of Cores 59-68, as their data are located mostly near the beginning of the evolution paths. Two groups of data can be considered, on either side of the vitrinite domain depending on their $\mathrm{H} / \mathrm{C}$ ratio. The first one is composed of points with low $\mathrm{H} / \mathrm{C}$ ratios ( 0.73 and less), near the limit of existence for kerogens and below that of vitrinite. Such material consists of degraded kerogen and is considered to be residual detritus (Tissot et al., 1979). It is representative of the low amounts of organic material $\left(0.3-0.8 \% \mathrm{C}_{\mathrm{org}}\right)$ in Unit 4 and in Cores 56-58 of Units 5 and 6 . The second group of kerogens shows higher $\mathrm{H} / \mathrm{C}$ ratios (1.08 and more) and lies between the Type II and III evolution paths. All the related samples belong to black material, rich in organic matter, with 5.3 to $6.3 \%$ organic carbon in the total rock. A predominantly aquatic origin (Type II) can be inferred for the kerogens of Cores 60 and 66 with the richest $\mathrm{H} / \mathrm{C}$ ratios (kerogen B1; Tissot et al., 1979). When only the $\mathrm{H} / \mathrm{C}$ ratio decreases (for example, Sample 511-68-2, 130-140 cm), we can deduce that organic material, either terrestrial (Type III; kerogen B2; Tissot et al., 1979) or residual, made a larger contribution. The only enrichment of the $\mathrm{O} / \mathrm{C}$ ratio, found for Sample 59-3, 79-82 cm when compared to Sample 511-59-3, 83-95 cm, implies another mechanism. A larger $\mathrm{O} / \mathrm{C}$ ratio was obtained for weathered samples compared to relatively well preserved samples; the weathering enriches the organic matter with oxygenated compounds at the expense of the hydrogenated ones. This 


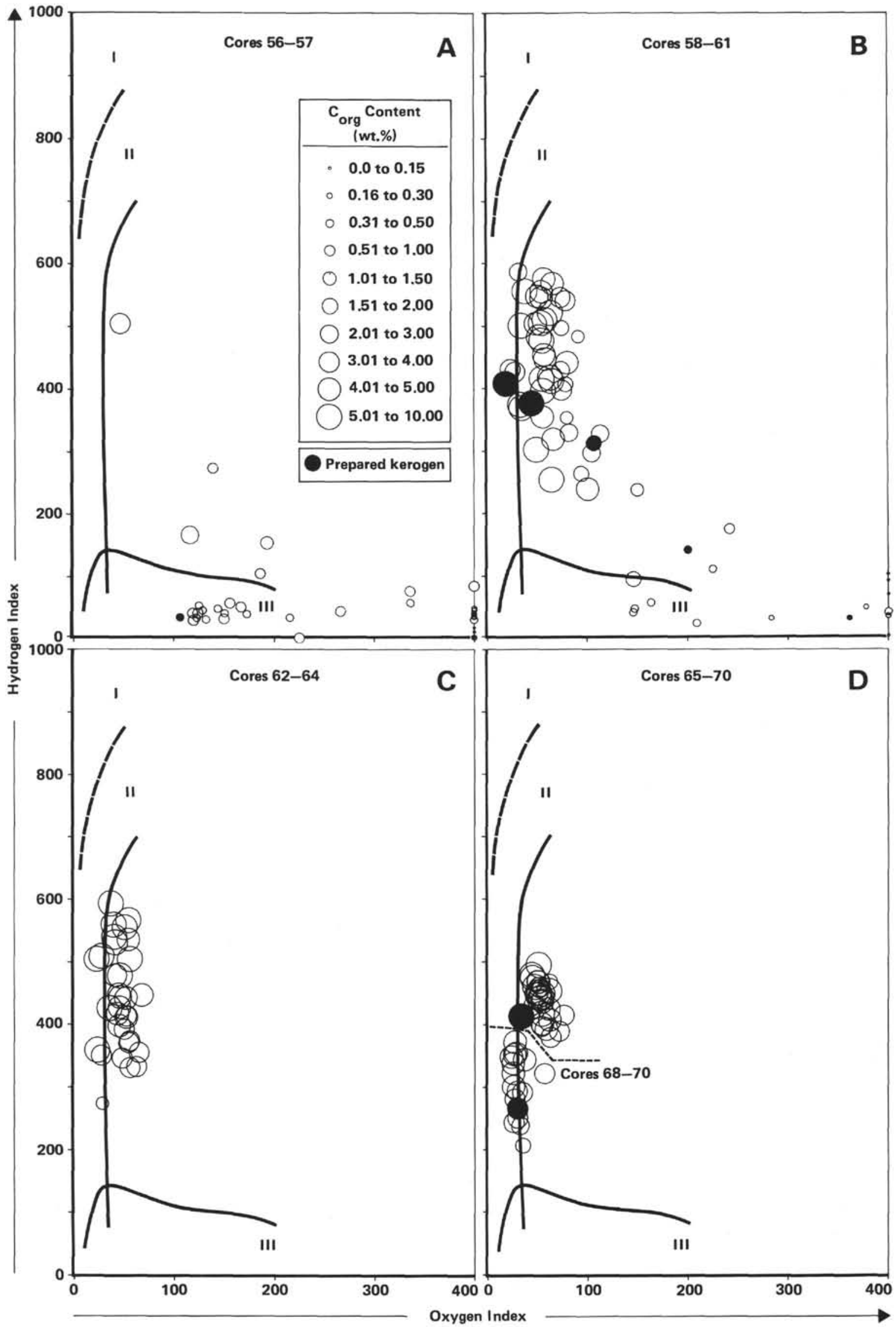

Figure 3. Pyrolysis assays: hydrogen and oxygen indexes, Hole 511. 


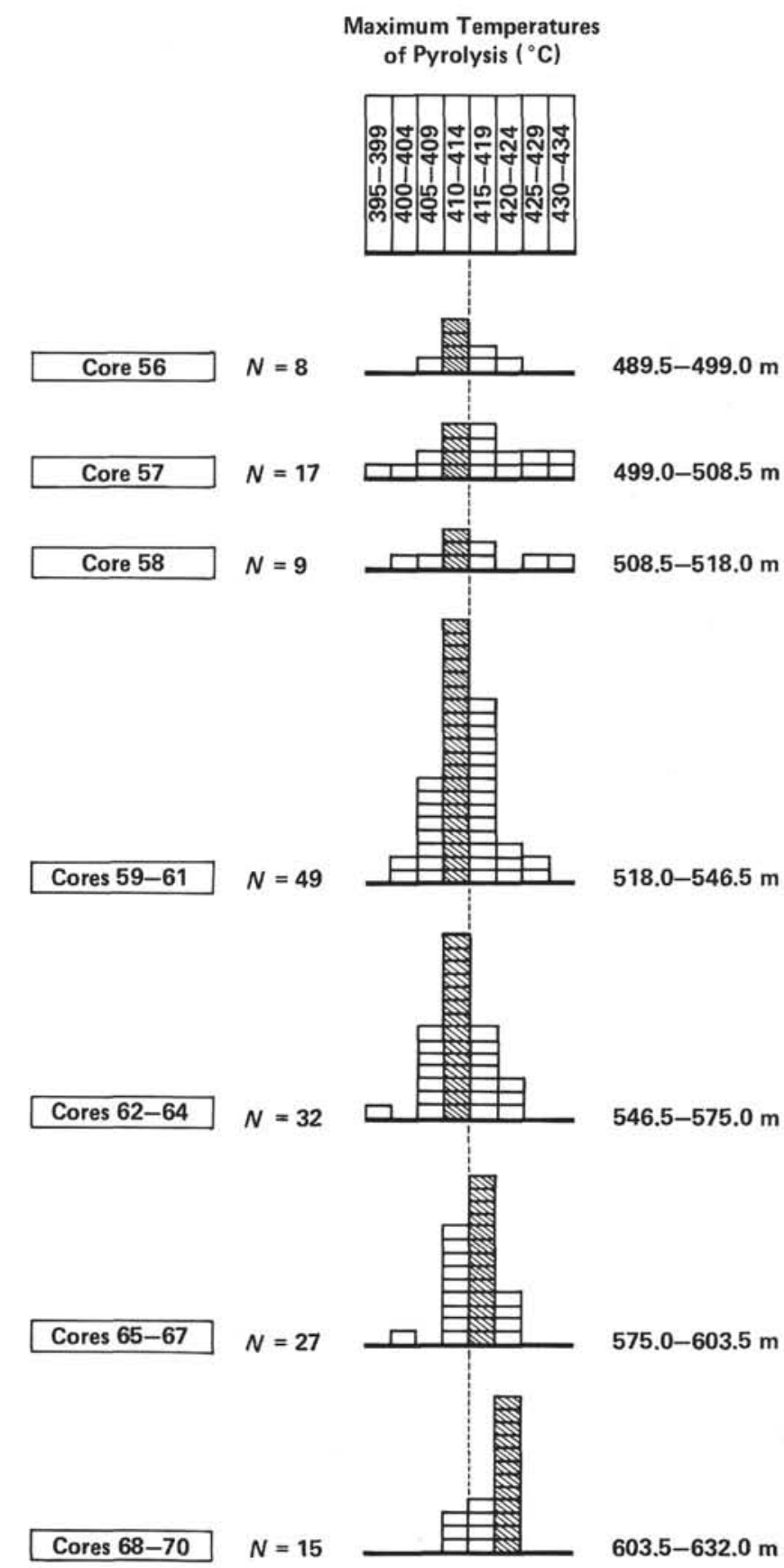

Figure 4. Pyrolysis assays: vertical distribution of maximum temperature classes, from Cores 56-70, Hole 511.

was also observed (Table 4) in the lower Toarcian of the Paris Basin for several samples from the same geological horizon, collected from outcrop to subsurface. The variation is obvious both for pyrolysis data on rock and for elemental analysis on related kerogen; it defines a so-called "alteration" path quite distinct from the evolution paths. That alteration trend, which is due to oxidation mechanisms, can be applied to the kerogen of Sample 511-59-3, 79-82 cm, compared to the other kerogens of Core 59 (Fig. 5).
Table 2. Organic carbon contents of humic compounds, Hole 511.

\begin{tabular}{|c|c|c|c|c|c|}
\hline \multirow[b]{2}{*}{$\begin{array}{l}\text { Core/Section } \\
\text { (interval in } \mathrm{cm} \text { ) }\end{array}$} & \multirow[b]{2}{*}{$\begin{array}{l}\text { Depth below } \\
\text { Seafloor } \\
\text { (m) }\end{array}$} & \multirow{2}{*}{$\begin{array}{l}\text { Total } \\
\text { Organic } \\
\text { Carbon }^{\mathrm{a}} \\
\text { (wt. \%) }\end{array}$} & \multicolumn{3}{|c|}{ Humic Compounds } \\
\hline & & & $\begin{array}{l}\text { Concentration } \\
\text { (ppm) }\end{array}$ & $\begin{array}{l}\text { Humic } \\
\text { Carbon } \\
\text { (wt. \%) }\end{array}$ & $\begin{array}{l}\text { Humic Carbon/ } \\
\text { Total Carbon }\end{array}$ \\
\hline $31-5,130-140$ & 259.30 & 0.51 & 55 & 0.06 & 11.8 \\
\hline $34-5,130-140$ & 287.80 & 0.69 & 40 & nsd & nsd \\
\hline $37-1,130-140$ & 310.30 & 0.82 & 70 & 0.07 & 8.5 \\
\hline $40-4,130-140$ & 343.30 & 0.62 & 70 & 0.07 & 11.3 \\
\hline $43-4,130-140$ & 371.80 & 0.65 & 35 & & \\
\hline $56-4,04-62$ & 493.54 & 0.44 & 25 & & \\
\hline $56-4,71-110$ & 494.21 & 0.13 & 12 & & \\
\hline $58-3,130-140$ & 512.80 & 0.31 & 25 & & \\
\hline $58-1,28-49$ & 513.28 & 0.38 & 10 & & \\
\hline $58-4,53-89$ & 513.53 & 0.13 & 20 & & \\
\hline $59-3,79-82$ & 521.79 & 0.48 & 10 & & \\
\hline $59-3,83-95$ & 521.83 & 5.75 & 45 & & \\
\hline $\begin{array}{l}59-3,98-110 \\
+59-4,21-22\end{array}$ & $\left.\begin{array}{l}521.98 \\
522.71\end{array}\right\}$ & 1.92 & 20 & & \\
\hline $60-5,130-140$ & 534.80 & 4.75 & 49 & & \\
\hline $62-1,96-134$ & 547.56 & 5.10 & 35 & & \\
\hline $62-5,130-140$ & 553.80 & 4.40 & 70 & 0.07 & 1.6 \\
\hline $64-4,130-140$ & 571.30 & 4.98 & 75 & 0.10 & 2.0 \\
\hline $65-2,78-109$ & 577.28 & 4.85 & 18 & & \\
\hline $66-4,130-140$ & 590.30 & 5.50 & 70 & 0.08 & 1.4 \\
\hline $68-2,130-140$ & 606.30 & 3.65 & 65 & 0.09 & 2.5 \\
\hline $70-3,130-140$ & 626.80 & 4.90 & 62 & 0.08 & 1.6 \\
\hline
\end{tabular}

Note: Blanks indicate nonsignificant data.

After $\mathrm{HCCl}_{3}$ extraction.

b Aumic compounds concentration of the analyzed solution.

Table 3. Elemental composition and ash content of kerogen, Hole 511.

\begin{tabular}{|c|c|c|c|c|c|c|c|c|c|c|}
\hline \multirow{2}{*}{$\begin{array}{c}\text { Core/Section } \\
\text { (interval in cm) }\end{array}$} & \multirow{2}{*}{$\begin{array}{l}\text { Depth below } \\
\text { Seafloor } \\
\text { (m) }\end{array}$} & \multicolumn{6}{|c|}{ Wt.\% on Ash-free Basis } & \multirow{2}{*}{$\begin{array}{c}\text { Ash } \\
\text { (wt. \%) }\end{array}$} & \multicolumn{2}{|c|}{ Atomic Ratio } \\
\hline & & c & $\mathrm{H}$ & $\mathrm{N}$ & 0 & s & $\mathrm{Fe}$ & & $\mathrm{H} / \mathrm{C}$ & $\mathrm{O} / \mathrm{C}$ \\
\hline $37-1,130-140$ & 310.30 & 34.8 & 2.0 & 1.4 & 12.6 & .23 .9 & 21.3 & 4.0 & 0.69 & 0.27 \\
\hline $56-4,4-62$ & 493.54 & 61.6 & 3.7 & 1.5 & 18.3 & 3.9 & 0.8 & 10.1 & 0.73 & 0.22 \\
\hline $58-3,130-140$ & 512.80 & 50.0 & 3.0 & 1.5 & 14.1 & 12.7 & 11.4 & 7,4 & 0.71 & 0.21 \\
\hline $59-3,79-82$ & 521.79 & 21.6 & 1.9 & 0.6 & 8.3 & 24.7 & 20.2 & 22.7 & 1.04 & 0.29 \\
\hline $59-3,83-95$ & 521.83 & 39.0 & 3.7 & 1.2 & 10.7 & 24.0 & 18.8 & 2.8 & 1.12 & 0.21 \\
\hline $\begin{array}{l}59-3,98-100 \\
+59-4,29-31\end{array}$ & $\left.\begin{array}{l}521.98 \\
522.71\end{array}\right\}$ & 38.4 & 3.5 & 1.6 & 9.6 & 22.5 & 19.3 & 5.2 & 1.09 & 0.19 \\
\hline $60-5,130-140$ & 534.80 & 46.0 & 4.8 & 1.3 & 11.2 & 20.8 & 15.6 & 0.5 & 1.24 & 0.18 \\
\hline $66-4,130-140$ & 590.30 & 49.1 & 4.9 & 1.5 & 9.5 & 19.1 & 13.7 & 2.4 & 1.19 & 0.15 \\
\hline $68-2,130-140$ & 606.30 & 46.7 & 4.0 & 1.6 & 11.5 & 18.6 & 14.5 & 3.0 & 1.02 & 0.18 \\
\hline
\end{tabular}

\section{Chloroform Extracts (Table 5, Fig. 6)}

All the samples selected yielded low amounts of extract (less than $50 \mathrm{mg}$ ) and low extract/organic carbon ratios (less than 0.02). Some of the extracts were too lean to be fractionated, so that extracts of two samples had to be combined.

Thin layer chromatography fractionation reveals a large fraction (67-77\%) of NSO compounds, and an aromatic $(12-19 \%)$ and a saturated + unsaturated fraction (11-14\%). The gas chromatography (GC) of the saturated fraction generally reveals a predominance of evencarbon-numbered molecules along the $n-\mathrm{C}_{15}-n-\mathrm{C}_{19}$ range (Fig. 6A) and an equal contribution of pristane and of phytane (Fig. 6A-B). Both characteristics are encountered in organic matter of marine origin, and the even predominance could be characteristic of reducing microenvironments (Welte and Ebhardt, 1968). A predominance of odd-carbon-numbered molecules is observed in the $n-\mathrm{C}_{23}-n-\mathrm{C}_{33}$ range. This would be indicative of an immature material derived from higher plants. In the same carbon-number range, cyclic molecules of the sterane and triterpane series should be present, as indicated by more detailed analyses (GC/MS) of other Cretaceous 


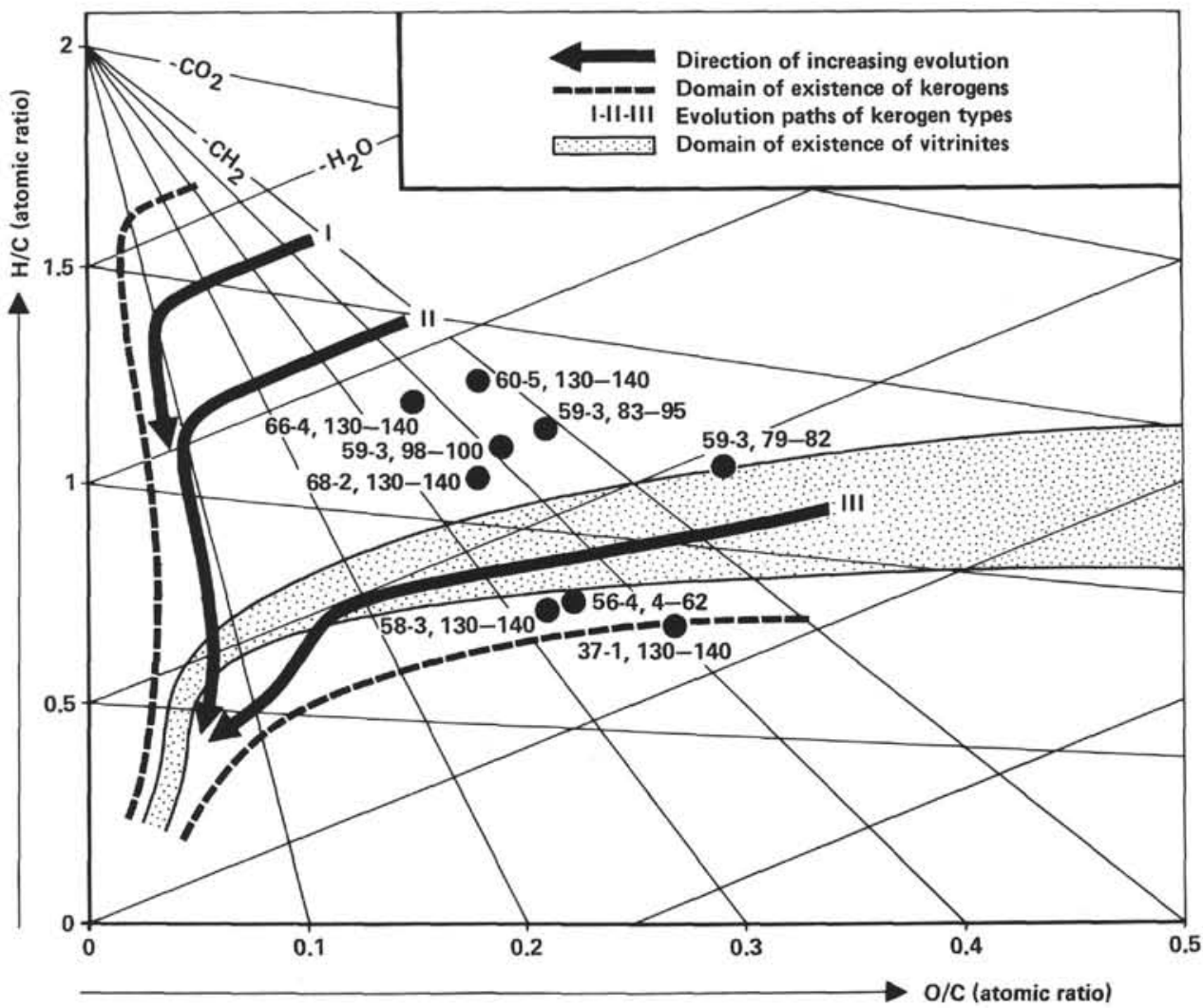

Figure 5. Kerogens: H/C versus $\mathrm{O} / \mathrm{C}$ diagram for Hole 511.

Table 4. Pyrolysis and elemental analysis data, lower Toarcian samples, Fécocourt, East Paris Basin.

\begin{tabular}{|c|c|c|c|c|c|c|c|c|c|c|c|}
\hline \multirow{3}{*}{$\begin{array}{l}\text { Subsurface } \\
\text { Depth } \\
\text { (m) }\end{array}$} & \multirow{3}{*}{$\begin{array}{l}\text { Total } \\
\text { Organic } \\
\text { Carbon } \\
\text { (wt.\%) }\end{array}$} & \multirow{3}{*}{$\begin{array}{l}\text { Pyrolysis } \\
\text { Hydrogen } \\
\text { Index }\end{array}$} & \multirow{3}{*}{$\begin{array}{l}\text { Rock-Eval } \\
\text { Oxygen } \\
\text { Index }\end{array}$} & \multicolumn{8}{|c|}{ Elemental Analysis, Kerogen Concentrate } \\
\hline & & & & \multicolumn{2}{|c|}{ Atomic Ratio } & \multicolumn{6}{|c|}{ Wt. \% on Ash-free Basis } \\
\hline & & & & $\mathrm{H} / \mathrm{C}$ & $\mathrm{O} / \mathrm{C}$ & C & H & $\mathrm{N}$ & 0 & S & $\mathrm{Fe}$ \\
\hline 0.5 & 2.0 & 13 & 238 & 1.18 & 0.23 & 40.66 & 47.96 & 0.64 & 9.54 & 1.20 & 0.81 \\
\hline 1.0 & 6.3 & 400 & 88 & 1.24 & 0.19 & 40.46 & 50.15 & 0.65 & 7.53 & 1.21 & 0.24 \\
\hline 3.8 & 8.1 & 529 & 65 & 1.29 & 0.15 & 40.30 & 51.86 & 0.74 & 5.96 & 1.14 & 0.94 \\
\hline 5.3 & 6.1 & 804 & 24 & 1.31 & 0.07 & 40.16 & 53.60 & 0.93 & 3.05 & 1.46 & 9.78 \\
\hline
\end{tabular}

Note: Pyrolysis data for rock, Bienner et al. (1977); elemental analysis data of related kerogen, Nicaise (1977).

Table 5. Composition of chloroform extracts, Hole 511.

\begin{tabular}{|c|c|c|c|c|c|c|c|}
\hline \multirow[b]{2}{*}{$\begin{array}{c}\text { Core/Section } \\
\text { (interval in cm) }\end{array}$} & \multirow[b]{2}{*}{$\begin{array}{l}\text { Depth below } \\
\text { Seafloor } \\
\text { (m) }\end{array}$} & \multirow[b]{2}{*}{$\begin{array}{l}\mathrm{HCCl}_{3} \text { Extract } \\
\text { (wt., mg) }\end{array}$} & \multirow[b]{2}{*}{ Extract/Rock } & \multirow[b]{2}{*}{ Extract/ $C_{\text {org }}$} & \multicolumn{3}{|c|}{$\begin{array}{l}\text { Thin Layer Chromatography } \\
\text { Fractionation }\end{array}$} \\
\hline & & & & & $\begin{array}{c}\text { NSO } \\
\text { Compounds } \\
(\%)\end{array}$ & $\begin{array}{l}\text { Aromatics } \\
(\%)\end{array}$ & $\begin{array}{l}\text { Sat. + Unsat. } \\
(\%)\end{array}$ \\
\hline $\begin{array}{l}60-5,130-140 \\
+62-5,130-140\end{array}$ & $\left.\begin{array}{l}534.8 \\
553.8\end{array}\right\}$ & 36.4 & 0.08 & 0.016 & 76 & 12 & 12 \\
\hline $\begin{array}{l}64-4,130-140 \\
+66-4,130-140\end{array}$ & $\begin{array}{l}571.3 \\
590.3\end{array} \mid$ & 39.0 & 0.08 & 0.016 & 67 & 19 & 14 \\
\hline $68-2,130-140$ & 606.3 & 31.5 & 0.06 & 0.017 & 77 & 12 & 11 \\
\hline $70-3,130-140$ & 626.8 & 48.2 & 0.10 & 0.029 & 72 & 15 & 13 \\
\hline
\end{tabular}

oceanic materials (Roucaché et al., 1979). Such compounds are more likely to be derived from marine material (Fig. 6B).

An unsaturated fraction is also present. GC analysis reveals compounds that are localized in the carbon-number range equivalent to $n-C_{15}-n-C_{19}$ and $n-C_{25}-n-C_{30}$ (Fig. 6C-D) but does not permit the identification of significant molecules.

\section{VERTICAL DISTRIBUTION OF ORGANIC MATTER}

Typical marine organic matter characterized the main part of Unit 6 from Samples 511-58-4, $105 \mathrm{~cm}$ to 511$70-5,54 \mathrm{~cm}$ (Fig. 3B-D), and the main range of the corresponding organic carbon in the total rock was 2-6 wt.\%. The lowest pyrolysis oxygen indexes ( 35 and less) 

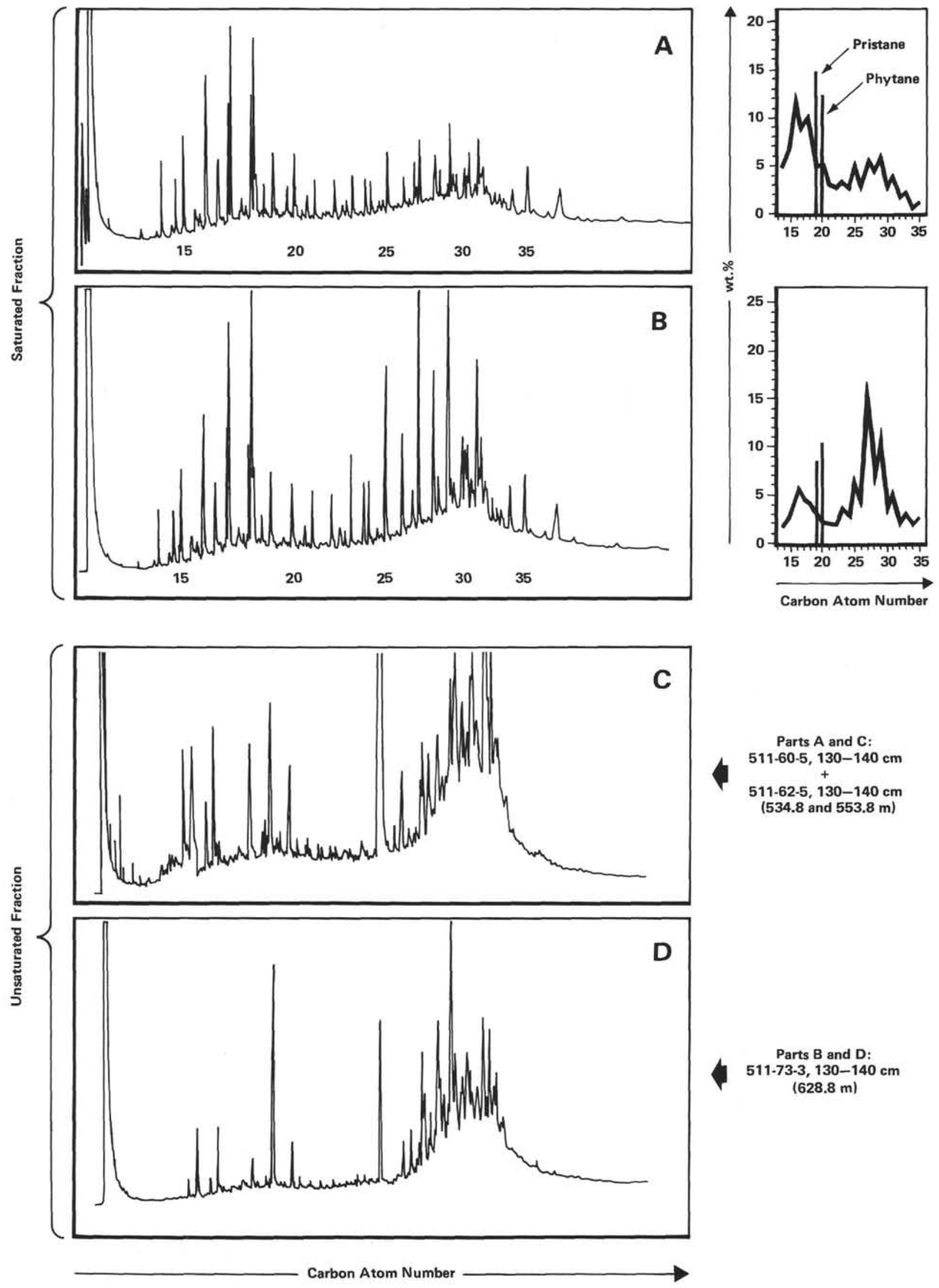

Parts $A$ and $C$ :

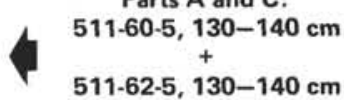
(534.8 and $553.8 \mathrm{~m}$ )

Parts B and D:

$511-73-3,130-140 \mathrm{~cm}$ $(628.8 \mathrm{~m})$

Figure 6. Extracts: gas chromatography of saturated and unsaturated hydrocarbons. GC analysis did not permit the identification of significant molecules of unsaturated hydrocarbons. 
are observed (Fig. 3D) for the bottom 23 meters in Cores $68-70$, when the fissile black material changes to a much softer one (Hole 511 site chapter, this volume). The major change for sedimentation between the muddy nannofossil chalks of Unit 5 and the black mudstones of Unit 6 is also observed for the organic matter. Upon the basis of pyrolysis data, the change characterizes a transitional zone (Fig. 7). The underlying material, which is rich in organic matter, is replaced by undifferentiated or residual detritus, where the organic carbon content is very minor and the hydrogen indexes low to null. Material from oxidizing environments characterizes a transitional zone from Sample 511-58-4, 87 $\mathrm{cm}$ to Sample 511-56-4, $4 \mathrm{~cm}$. Reducing environmental conditions also recur in the middle part of this zone (511-57-6, $6 \mathrm{~cm}$ to $511-57-2,95 \mathrm{~cm})$, where a more or less altered marine organic matter is interlain with residual detritus. Some reducing conditions temporarily reappear among oxidizing ones in the upper part of the zone and preserve the terrigenous component of the organic material, that is, the detritus. Such a detrital material is found from $511-57-2,35 \mathrm{~cm}$ to $511-56-4,4 \mathrm{~cm}$ and defines the finely laminated black shales, interlain at the bottom of Core 56 with muddy nannochalks and black zeolitic clays. Residual organic matter characterizes the latter two facies. Above the transitional zone, an oxidizing environment prevails from Sample 511-56-3, of Unit 5 throughout Unit 4 . This major change corresponds to samples with a very low content of organic matter that are devoid of hydrocarbons upon pyrolysis and are designated here as "inert" residual organic matter (Fig. 7).

In summary, the vertical distribution of organic matter along the Upper Jurassic-Cretaceous sequence of Site 511 reveals a transitional zone about 20 meters thick at the top of the black shales and mudstones of Unit 6 and at the bottom of the muddy nannofossil chalks of the adjacent Unit 5. Residual organic matter indicative of oxidizing conditions occurs throughout this transitional zone and prevails along the overlying layers of Units 4 and 5. Black shale facies in the middle part of the transitional zone reveal marine organic matter, implying the reducing environment that was found for the main part of Unit 6, and detrital, nonmarine organic matter deposited in a less reducing environment characterizes the same black facies at the bottom of Unit 5 .

\section{GEOCHEMICAL RELATIONSHIP OF SITE 511 WITH SITES 327 AND 330}

The organic matter zonation during the Late Jurassic-Cretaceous for Site 511 may be compared with that of the adjacent Sites 327 and 330 (Table 6).

In Hole 327A, 45 samples had been studied from Cores 10 to 27 (Herbin and Deroo, 1979), dating from the late Maestrichtian and Aptian to Neocomian(?); from Hole 330, 71 samples were taken in Cores 1-16, between early-middle Albian and Oxfordian-Middle Jurassic(?).

The three sites, located very close to each other (Fig. 1), show identical fluctuations of the sedimentological environment between the Albian and Upper Jurassic, especially for the transitional zone between the reducing and oxidizing environments (Table 6). This transitional zone is well defined at Site 511 from Samples 511-58-4, $89 \mathrm{~cm}$ to $511-56-4,4 \mathrm{~cm}$ and is present in Hole 327A, Cores 22 and 23. Furthermore, some dilution of the marine organic matter by detrital material appears at the bottom of Unit 6 in Hole 511. The same change is observed in Hole 330, from Section 330-8-4 to Core 10.

When considered as a whole, these relationships for the three sites allow us to define the paleographical outlines of the eastern Falkland Plateau:

1) During Middle Jurassic time, detrital sediments (sandstone, siltstone) and detrital organic matter issuing from the American and possibly African continents were deposited (bottom sediments of Hole 330).

2) The widespread Callovian-Oxfordian transgression allowed the formation of the young South Atlantic Basin. Existence of barriers prevented water exchange but induced a water stratification, which in turn resulted in anoxic environments and consequently good preservation of the marine organic matter. Such conditions prevailed until the early Albian.

3) At that time, the barriers broke, owing to the drifting phase. The opening allowed progressively more oxygenated waters to invade the South Atlantic Basin, converting the reducing to an oxidizing environment. The transitional zone which separates the two characteristic environments extends over some 20 meters and represents a period of several million years. Various types of organic matter-unaltered and altered marine residual, and detrital-coexist in this transitional zone depending on the level sampled.

4) During the period following the Aptian, oxidizing environments and residual organic matter prevailed. This is indicative of a deep alteration in the nature of the organic matter from both marine and detrital sources. Thus definite submersion of the barriers by oxygenated currents and a complete oceanization of the southern part of the South Atlantic can be inferred.

The previous study in the South Atlantic Basin (Herbin and Deroo, 1979) showed that the same environments existed after the early Aptian at Site 361 (Leg 40). In this case, the transitional zone between the reducing and oxidizing environments was located between Cores 27 and 28 , Hole 361 .

In order to make a more accurate paleogeographic sketch, particularly during the breakup of the eastern Falkland Plateau, it would be necessary to study in great detail the cores from the transitional zone which separates the reducing and oxidizing environments (equivalent to Cores 56 to 59 in Hole 511) for Sites 327 and 330.

\section{CONCLUSION}

Organic matter present in the Upper Jurassic-Cretaceous sediments of Site 511 belongs to two main types: (1) material of marine origin, rich in organic matter, which implies reducing depositional environments; (2) a very lean residual material low in organic matter and related to oxidizing environmental conditions. Pyrolysis and kerogen analyses clearly defined these two types of material, and hydrocarbon analyses confirmed the reducing conditions for the marine one. An immature 


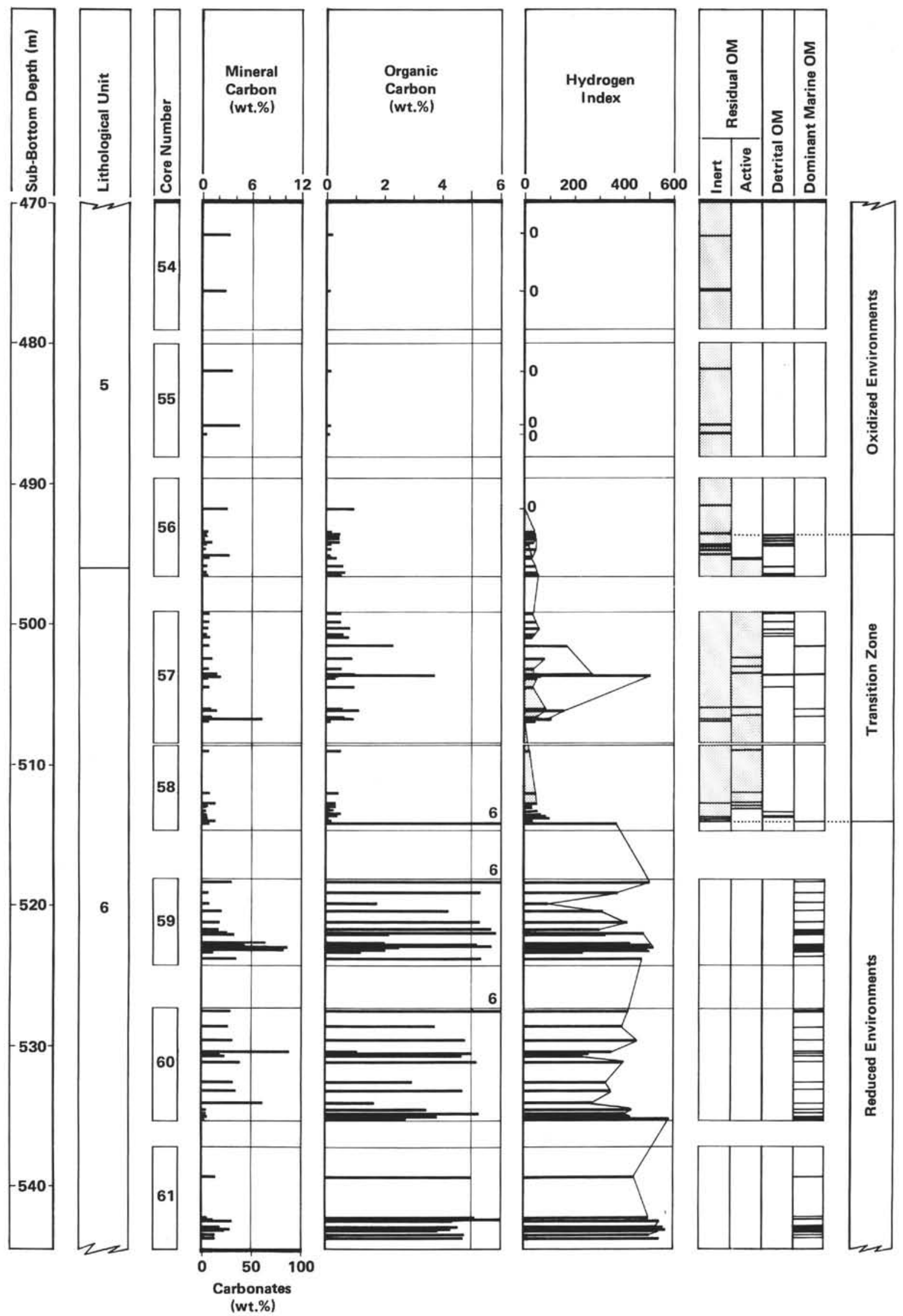

Figure 7. Vertical distribution of organic matter (OM) in Cores 54 to 61 at the limit of Lithological Units 5 and 6 , Hole 511. 
Table 6. Zonation of organic matter in Cretaceous and Jurassic sediments, Holes 327A, 330, and 511.

\begin{tabular}{|c|c|c|c|}
\hline & Hole 327A & Hole 330 & Hole 511 \\
\hline \multicolumn{4}{|l|}{ Oceanization with currents } \\
\hline $\begin{array}{l}\text { Oxidized Environments } \\
\text { Residual (inert) organic } \\
\text { matter, } C_{\text {org }}=0.1-0.6 \%\end{array}$ & $\begin{array}{l}\text { Cores 20-21: early to } \\
\text { middle Albian. } \\
\text { Unit 7: Bioturbated vari- } \\
\text { colored nannofossil ooze } \\
\text { to chalk or claystone. }\end{array}$ & $\begin{array}{l}\text { Cores 1-2: early to middle } \\
\text { Albian. } \\
\text { Unit } 2: \text { Light brown and } \\
\text { pinkish gray bands of } \\
\text { zeolite-rich nanno- } \\
\text { fossil clay. }\end{array}$ & $\begin{array}{l}\text { Core } 50 \text { to Sample } 511-56- \\
3,145 \mathrm{~cm} \text { : middle Albian. } \\
\text { Unit } 5 \text { : Variegated clay- } \\
\text { stone and muddy nanno- } \\
\text { fossil chalks. }\end{array}$ \\
\hline $\begin{array}{l}\text { Transition Zone } \\
\text { Residual, detrital or } \\
\text { aquatic organic matter } \\
C_{\text {org }}=0.1-3.7 \%\end{array}$ & $\begin{array}{l}\text { Cores 22-23: middle to late } \\
\text { Aptian. } \\
\text { Unit } 8 \text { : Brown black to } \\
\text { olive gray claystone. }\end{array}$ & $\begin{array}{l}\text { ( } 38 \mathrm{~m} \text { between Cores } 2 \\
\text { and } 3 \text { ) }\end{array}$ & $\begin{array}{l}\text { Sample } 511-56-4,4 \mathrm{~cm} \text { to } \\
\text { Sample } 511-58-4,87 \mathrm{~cm} \text { : } \\
\text { early Albian to early } \\
\text { Aptian. } \\
\text { Unit 6: Black mudstone } \\
\text { and nannofossil mud. } \\
\text { stone (thickness }=20 \mathrm{~m} \text { ). }\end{array}$ \\
\hline \multicolumn{4}{|c|}{ Oceanization without currents $=$ water stratification } \\
\hline Reduced Environments & $\begin{array}{l}\text { Core 24-27: Aptian to } \\
\text { Neocomian(?). }\end{array}$ & $\begin{array}{l}\text { Core 3-Sample 330-8-3, } \\
55 \mathrm{~cm} \text { : Aptian to } \\
\text { Oxfordian. }\end{array}$ & $\begin{array}{l}\text { Sample } 511-58-4,105 \mathrm{~cm} \text { to } \\
\text { Sample } 511-68-2,58 \mathrm{~cm} \text { : } \\
\text { carly Aptian to Late } \\
\text { Jurassic: }\end{array}$ \\
\hline $\begin{array}{l}\text { Aquatic organic matter } \\
C_{\text {org }}=1-6 \%\end{array}$ & $\begin{array}{l}\text { Unit 8: Brownish black } \\
\text { to olive gray claystone } \\
\text { rich in organic matter, } \\
\text { interbedded with } \\
\text { occasional greenish gray, } \\
\text { thick, micritic limestone } \\
\text { layers. }\end{array}$ & $\begin{array}{l}\text { Unit 3: Olive black } \\
\text { carbonaceous claystone. }\end{array}$ & Unit 6: (contd.). \\
\hline $\begin{array}{l}\text { Aquatic + detrital organic } \\
\text { matter } \\
C_{\text {org }}=1.6 / 4.9 \%\end{array}$ & & $\begin{array}{l}\text { Sample } 330-8-4,104 \mathrm{~cm} \text { to } \\
\text { Section 330-10-1: } \\
\text { Oxfordian. }\end{array}$ & $\begin{array}{l}\text { Sample } 511-68-2,130 \mathrm{~cm} \text { to } \\
\text { Core 70: Late Jurassic }\end{array}$ \\
\hline $\begin{array}{l}\text { Detrital organic matter } \\
\mathrm{C}_{\text {org }}=0.1-2.6 \%\end{array}$ & & $\begin{array}{l}\text { Unit 3: (contd.). } \\
\text { Section 330-10-2-Core 16: } \\
\text { Oxfordian-Middle } \\
\text { Jurassic? } \\
\text { Units 4-6: Silty clay and } \\
\text { clayey silt; arkosic } \\
\text { sandstone. }\end{array}$ & Unit 6: (contd.). \\
\hline
\end{tabular}

stage of evolution can be assigned to all the samples studied.

The vertical distribution of the various types of organic matter, based on numerous samplings, reveals a transitional zone where the black mudstones of Unit 6 pass to the zeolitic clays and claystones of Unit 5. This transitional zone was also defined from the adjacent Hole 327A and should be present in Hole 330. Thus, a geographical outline can be proposed for Mesozoic times in the eastern Falkland area, based on the distribution of the various types of organic matter at the three DSDP sites.

\section{ACKNOWLEDGMENTS}

The authors are indebted to Dr. B. Simoneit and Dr. H. von der Dick for comments and review of the present paper.

\section{REFERENCES}

Espitalié, J., Laporte, J. L., Madec, M., Marquis, F., Leplat, P., Paulet, J., and Boutefeu, A., 1977. Méthode rapide de caractérisation des roches mères, de leur potentiel pétrolier et de leur degré d'évolution. Rev. Inst, Fr. Pet., 32:23-42.
Herbin, J. P., and Deroo, G., 1979. Etude sédimentologique de la matière organique dans les argiles noires crétacées de l'Atlantique Sud. Doc. Lab. Géol. Fac. Sci. Lyon, No. 75, pp. 71-87.

Huc, A. Y., Durand, B., and Monin, J. C., 1978. Humic compounds and kerogen in cores from Black Sea sediments, Leg 42B-Holes 379A, B, and 380A. In Ross, D. A., Neprochnov, Y. P., et al., Init. Repts. DSDP, 42, Pt. 2: Washington (U.S. Govt. Printing Office), 737-748.

Roucaché, J., Deroo, G., and Boulet, R., 1979. Caractérisation par différentes méthodes physico-chimiques de types de matière organique dans les sédiments du Crétacé d'Atlantique en mer profonde. Rev. Inst. Fr. Pet., 34(2):191-220.

Tissot, B., Deroo, G., and Herbin, J. P., 1979. Organic matter in Cretaceous sediments of the North Atlantic: Contribution to sedimentology and paleogeography. In Talwani, M., Hay, W., and Ryan, W. B. F. (Eds.), Deep Drilling Results in the Atlantic Ocean: Continental Margins and Paleoenvironment. Am. Geophys. Union, Maurice Ewing Series 3:362-374.

Tissot, B., Durand, B., Espitalié, J., and Combaz, A., 1974. Influence of the nature and diagenesis of organic matter in the formation of petroleum. Bull. Am. Assoc. Pet. Geol., 58:499-506.

Welte, D. H., and Ebhardt, G., 1968. Distribution of long chain $n$ paraffins and fatty acids in sediments from the Persian Gulf. Geochim. Cosmochim. Acta, 32:465-466. 